\title{
Iperparatiroidismo primitivo in paziente con rachitismo ipofosforemico
}

\author{
F. Rainone ${ }^{1}$, T. Arcidiacono ${ }^{1}$, S. Capelli ${ }^{2}$, V. Donghi ${ }^{3}$, M. Di Frenna ${ }^{3}$, S. Mora ${ }^{2}$, \\ G. Weber ${ }^{3}$, G. Vezzoli ${ }^{1}$
}

${ }^{1}$ Unità di Nefrologia e Dialisi, Istituto Scientifico San Raffaele, Università Vita Salute, Milano

${ }^{2}$ Laboratorio di Endocrinologia Pediatrica, Bonetwork, Istituto Scientifico San Raffaele, Milano

${ }^{3}$ Divisione di Pediatria, Istituto Scientifico San Raffaele, Università Vita Salute, Milano

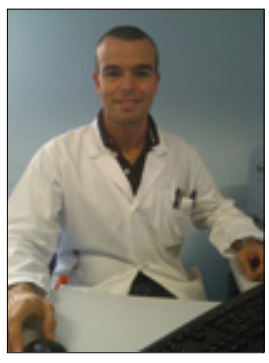

Francesco Rainone

\section{Introduzione}

Il rachitismo ipofosforemico comprende un gruppo di patologie rare ereditarie caratterizzate da un deficit di riassorbimento renale del fosfato, ipofosfatemia e deformazioni ossee $(1,2)$. Le forme più comuni sono il rachitismo ipofosforemico a trasmissione X-linked dominante e il rachitismo ipofosforemico autosomico dominante (AD) $(3,4)$. Le altre forme sono molto più rare e sono riassunte nella Tabella I (5-15). La forma X-linked è dovuta alle mutazioni del gene PHEX (cromosoma

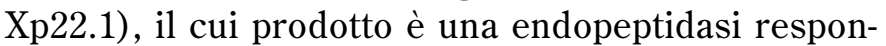
sabile della inattivazione della fosfatonina FGF23 (fibroblast growth factor 23), che da esso viene lisata in due frammenti inattivi $(3,4)$. Il rachitismo ipofosforemico $\mathrm{AD}$ è causato invece da mutazioni che interessano direttamente il gene FGF23 (cromosoma 12p13.3) e che rendono il suo prodotto insensibile all'azione proteolitica di PHEX (4). Queste forme di rachitismo si caratterizzano perciò per un aumento delle concentrazioni seriche di FGF23 (16).

FGF23 è un ormone sintetizzato principalmente dagli osteociti e dagli osteoblasti e che regola l'omeostasi del fosfato agendo a più livelli (16). Nel tubulo renale prossimale riduce il riassorbimento del fosfato perché inibisce l'espressione dei carrier del fosfato NPT2a e NPT2c sulla membrana luminale dei tubulociti. FGF23 riduce così la soglia di escrezione del fosfato e contribuisce in modo sostanziale alla regolazione della fosforemia. L'anomalo aumento dei suoi livelli circolanti nei pazienti con rachitismo ipofosforemico X-linked e $\mathrm{AD}$ fa sì che il tubulo renale sia incapace di mantenere la fosforemia entro il range di norma. FGF23 inibisce inoltre l'attività della 1-alfa-idrossilasi renale, responsabile della attivazione del $25 \mathrm{OHD}$ a $1,25(\mathrm{OH})_{2} \mathrm{D}$, e attraverso questo meccanismo riduce indirettamente l'assorbimento intestinale del fosfato (16).

Da un punto di vista biochimico i pazienti affetti da rachitismo ipofosforemico presentano ipofosforemia, normale calcemia, valori plasmatici di PTH normali o lievemente elevati, livelli circolanti di $1,25(\mathrm{OH})_{2} \mathrm{D}$ normali o relativamente ridotti e livelli serici di fosfatasi alcalina aumentati o normali (negli adulti). Da un punto di vista clinico, i pazienti manifestano un quadro di rachitismo caratterizzato da deficit della crescita e deformità scheletriche di grado moderato o severo che esordiscono nella prima infanzia in concomitanza con l'inizio della deambulazione. Le deformità più comuni comprendono varismo o valgismo degli arti inferiori, deformazione a sciabola delle tibie, coste a corona di rosario, allargamento delle epifisi (3). I pazienti possono riferire anche astenia e una parte di essi sviluppano problemi muscolari severi attribuiti al deficit di fosfato e di vitamina $\mathrm{D}$ (17).

Il trattamento precoce con calcitriolo e fosfati ha permesso di ottenere buoni outcome clinici in termini di crescita e prevenzione delle deformità ossee (18). Tuttavia questa terapia non è scevra da complicanze perché la rapida escrezione urinaria che segue alla supplementazione orale di fosfato espone al rischio 
di nefrocalcinosi e calcolosi renale. Inoltre l'apporto di fosfato può stimolare la secrezione di PTH che insieme alla carenza di $1,25(\mathrm{OH})_{2} \mathrm{D}$ può giustificare lo sviluppo di iperplasia paratiroidea $(19,20)$. Questa si presenta usualmente come iperplasia diffusa, ma occasionalmente si manifesta con adenomi (21, 22). Il controllo ottimale della funzione paratiroidea è perciò complesso e lo stimolo cronico alla secrezione di PTH può causare lo sviluppo di iperparatiroidismo secondario e autonomo.

I benefici ottenuti dalla terapia con fosfato e calcitriolo sulla crescita e sullo sviluppo scheletrico ne giustificano l'uso nei bambini, mentre è discussa l'opportunità di continuare tale trattamento nell'individuo adulto dopo la fine dell'accrescimento. L'adulto resta esposto al rischio di osteomalacia secondaria alla ipofosforemia, ma l'assunzione della terapia con fosfato e vitamina $\mathrm{D}$ sottopone al rischio delle citate complicanze paratiroidee e renali. Molti Centri preferiscono perciò interrompere la terapia al termine della crescita. Il caso clinico qui descritto propone queste problematiche e le difficoltà nel bilanciare gli effetti terapeutici con i rischi di complicanze.

\section{Presentazione del Caso}

L.P. è una donna nata nel 1983, con una diagnosi clinica e biochimica di rachitismo ipofosforemico. Questa diagnosi fu posta all'età di 2 anni a seguito della comparsa di valgismo degli arti inferiori, deficit della crescita e ritardo nella dentizione decidua (13 mesi), associati a ipofosforemia e bassa soglia di escrezione dei fosfati. Nell'anamnesi familiare era riferita bassa statura e varismo degli arti inferiori nelle zie del ramo materno. I geni PHEX, FGF23 e DMP1 (Tab. I) sono stati testati, ma non sono state rilevate mutazioni della loro sequenza nucleotidica. La negatività dell'analisi genetica non consente un inquadramento nosologico preciso, ma il possibile sviluppo della malattia nelle sorelle della madre, orienta verso una forma di rachitismo ipofosforemico AD.

La paziente è stata inizialmente seguita presso gli ambulatori specialistici pediatrici. Dopo la diagnosi la paziente è stata posta in terapia con $1,25(\mathrm{OH})_{2} \mathrm{D}$ (dose media $40 \mathrm{ng} / \mathrm{kg} / \mathrm{die}$ ) e fosfati (dose media $2 \mathrm{~g} / \mathrm{m}^{2} /$ die) a dosi progressivamente crescenti per essere adeguate allo sviluppo corporeo. $\grave{E}$ stata anche associata una supplementazione con carbonato di calcio in relazione alle concentrazioni plasmatiche di PTH. Nonostante la terapia, i valori della fosforemia erano spesso inferiori ai valori di norma, i livelli della fosfatasi alcalina erano elevati e le concentrazioni di PTH erano ai valori alti di norma.

All'età di 11 anni (1994) sono state riscontrate calcificazioni para-articolari alle mani e ai piedi attribuite ad entesite calcifica, mentre l'esame ecografico dei reni ha dimostrato iperriflettività delle piramidi renali, compatibile con iniziale nefrocalcinosi. Dall'età di 18 anni (2001) la paziente è stata sottoposta a plurimi interventi correttivi ortopedici di allungamento delle tibie e dei femori. Nel 2005 è stata diagnosticata una steno-insufficienza aortica di grado lieve-moderato.

All'età di 24 anni, nel luglio 2007, la paziente è stata affidata alle cure del nefrologo. Era in terapia con calcitriolo $0,5 \mathrm{mcg}$ die e calcio carbonato $1 \mathrm{~g}$ die per os e gli esami dimostravano normali concentrazioni plasmatiche di calcio e PTH con ipofosforemia e livelli serici di $25(\mathrm{OH}) \mathrm{D}$ normali, ma inappropriati in rapporto ai livelli di PTH (Tab. II). Al successivo controllo del novembre 2007 veniva osservato un incremento della calcemia, per il quale veniva sospeso il calcio carbonato dalla terapia, ridotto il calcitriolo a $0,25 \mathrm{mcg} / \mathrm{die}$ e iniziato trattamento con colecalciferolo $500 \mathrm{U} /$ die.

Agli accertamenti dell'aprile 2009, la paziente presentava un ulteriore aumento della calcemia e PTH plasmatico elevato. Veniva pertanto sospesa la terapia con calcitriolo ed eseguita ecografia del collo, che mostrava un'immagine nodulare ipoecogena delle dimensioni di 9x6x4 mm compatibile con tumefazione paratiroidea. La scintigrafia paratiroidea con MIBI e pertecnetato era negativa per accumuli riferibili a paratiroide. Nonostante quest'ultimo dato, il quadro clinico era compatibile con iperparatiroidismo primitivo e veniva proposto l'intervento chirurgico di exeresi del nodulo paratiroideo, che tuttavia la paziente rifiutava. Veniva pertanto iniziata nel luglio 2009 terapia con il calciomimetico cinacalcet alla dose iniziale di $15 \mathrm{mg}$ e poi di $30 \mathrm{mg}$ bid. Dall'ottobre 2009 la dose di cinacalcet veniva aumentata a $60 \mathrm{mg}$ bid ed era associata alla somministrazione di calcitriolo e colecalciferolo. A seguito di queste modifiche terapeutiche i livelli plasmatici di PTH e calcio diminuivano, mentre quelli di fosforemia aumentavano soprattutto nei primi mesi di trattamento (Tab. II). Nel marzo 2010 venivano sospesi cinacalcet e calcitriolo per una rivalutazione del quadro clinico e si assisteva a una risalita dei valori plasmatici di PTH e calcio (Tab. II). Il controllo MOC total body mostrava la normalità dei valori di BMD come già riscontrato ai precedenti controlli, eseguiti da quando la paziente aveva 10 anni di età (Tab. III). L'ecografia del collo era invariata a distanza di un anno dalla precedente. 
TABELLA I - CARATTERISTICHE DELLE MALATTIE MONOGENICHE CON DISTURBO DEL RIASSORBIMENTO TUBULARE DEL FOSFATO E RACHITISMO

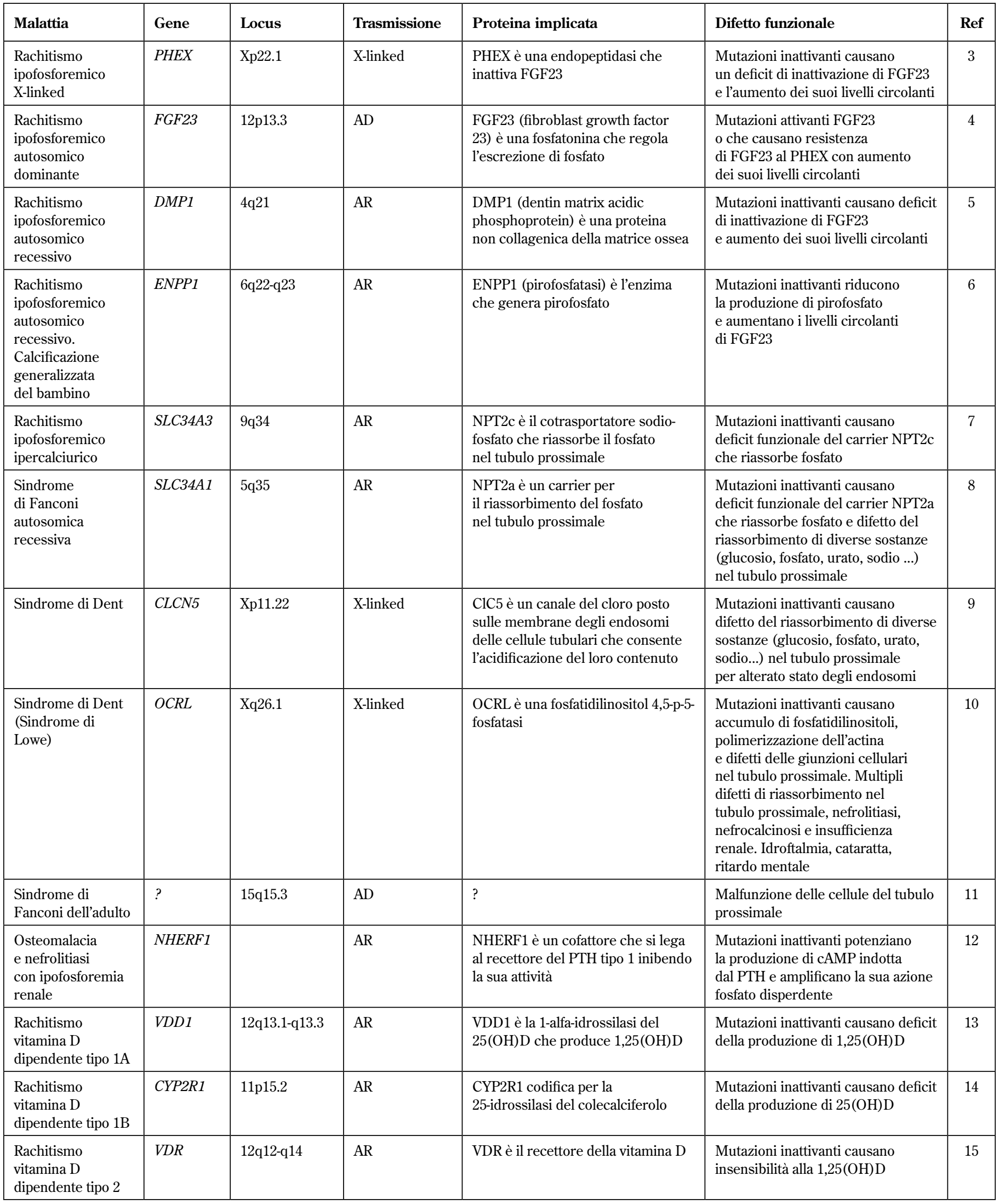


TABELLA II - EVOLUZIONE DELLE DIVERSE VARIABILI BIOUMORALI MISURATE NELLA PAZIENTE DURANTE IL FOLLOWUP IN RELAZIONE ALLE DIVERSE TERAPIE INTRAPRESE. LA TERAPIA CON CINACALCET ERA COMINCIATA NEL LUGLIO 2009 E CONTINUATA FINO AL MARZO 2010 (TRA PARENTESI SONO RIPORTATI I VALORI NORMALI DELLE DIVERSE VARIABILI)

\begin{tabular}{|c|c|c|c|c|c|c|c|c|c|c|c|c|}
\hline & $\begin{array}{l}\text { Luglio } \\
2007\end{array}$ & $\begin{array}{c}\text { Novembre } \\
2007\end{array}$ & $\begin{array}{l}\text { Luglio } \\
2008\end{array}$ & $\begin{array}{l}\text { Aprile } \\
2009\end{array}$ & $\begin{array}{l}\text { Giugno } \\
2009\end{array}$ & $\begin{array}{c}\text { Ottobre } \\
2009\end{array}$ & $\begin{array}{c}\text { Novembre } \\
2009\end{array}$ & $\begin{array}{c}\text { Gennaio } \\
2010\end{array}$ & $\begin{array}{c}\text { Marzo } \\
2010\end{array}$ & $\begin{array}{l}\text { Aprile } \\
2010\end{array}$ & $\begin{array}{c}\text { Settembre } \\
2010\end{array}$ & $\begin{array}{c}\text { Gennaio } \\
2011\end{array}$ \\
\hline $\begin{array}{l}\text { Fosforemia } \\
(0.8-1.5 \mathrm{~mol} / \mathrm{l})\end{array}$ & 0.45 & 0.58 & 0.45 & 0.4 & 0.58 & 0.71 & 0.77 & 0.55 & 0.65 & 0.45 & 0.52 & 0.53 \\
\hline $\begin{array}{l}\text { Calcemia } \\
(2.2-2.6 \mathrm{mmol} / \mathrm{l})\end{array}$ & 2.48 & 2.55 & 2.6 & 2.63 & 2.6 & 2.25 & 2.18 & 2.1 & 2.25 & 2.43 & 2.55 & 2.42 \\
\hline $\begin{array}{l}\text { Vitamina D } \\
(10-66 \mathrm{ng} / \mathrm{ml})\end{array}$ & 19.5 & & 15.9 & 12.5 & & 24.8 & & & 24.8 & & 60.4 & \\
\hline $\begin{array}{l}\text { Fosfatasi alcalina } \\
(56-119 \mathrm{U} / \mathrm{l})\end{array}$ & 83 & & & & & 114 & & & 114 & & 114 & \\
\hline $\begin{array}{l}\text { Fosfaturia } \\
(10-32 \mathrm{mmol} / 24 \mathrm{~h})\end{array}$ & 25.9 & 19.87 & 13.74 & 14.93 & & & 28.68 & & & & & \\
\hline $\begin{array}{l}\text { Terapia } \\
\text { - } 1,25(\mathrm{OH}) \mathrm{D}(\mathrm{mcg}) \\
\text { - CaCO3 }(\mathrm{g}) \\
\text { - Colecalciferolo }(\mathrm{U}) \\
\text { - Cinacalcet (mg) }\end{array}$ & $\begin{array}{c}0.5 \\
1\end{array}$ & $\begin{array}{c}0.5 \\
1\end{array}$ & $\begin{array}{l}0.25 \\
500\end{array}$ & $\begin{array}{l}0.25 \\
500\end{array}$ & 500 & $\begin{array}{c}750 \\
30\end{array}$ & $\begin{array}{c}0.5 \\
750 \\
60\end{array}$ & $\begin{array}{c}0.5 \\
750 \\
60\end{array}$ & $\begin{array}{c}0.5 \\
750 \\
60\end{array}$ & 1000 & 1000 & 1000 \\
\hline
\end{tabular}

TABELLA III - VALORI DI DENSITÀ MINERALE OSSEA RILEVATI ALLE SEDI VERTEBRALI E DEL COLLO FEMORALE MEDIANTE METODICA DXA. IL T-SCORE Ė IL NUMERO DI DEVIAZIONI STANDARD DALLA MEDIA DELLA POPOLAZIONE GIOVANE DELLO STESSO SESSO AL PICCO DI MASSA OSSEA ED Ė STATO PERCIÒ CALCOLATO SOLO NELLE MISURAZIONI DI DENSITÀ OSSEA SUCCESSIVE AI 20 ANNI

\begin{tabular}{|c|c|c|c|c|c|c|c|}
\hline & $\begin{array}{c}1993 \\
10 \text { anni }\end{array}$ & $\begin{array}{c}1994 \\
11 \text { anni }\end{array}$ & $\begin{array}{c}1995 \\
12 \text { anni }\end{array}$ & $\begin{array}{c}2001 \\
18 \text { anni }\end{array}$ & $\begin{array}{c}2004 \\
21 \text { anni }\end{array}$ & $\begin{array}{c}2009 \\
26 \text { anni }\end{array}$ & $\begin{array}{c}2010 \\
27 \text { anni }\end{array}$ \\
\hline $\begin{array}{l}\text { Lombare L1-L4 } \\
\mathrm{g} / \mathrm{cm}^{2} \\
\text { T-score }\end{array}$ & $\begin{array}{c}0.805 \\
-\end{array}$ & $\begin{array}{c}0.849 \\
-\end{array}$ & $\begin{array}{c}1.088 \\
-\end{array}$ & $\begin{array}{c}1.189 \\
-\end{array}$ & $\begin{array}{c}1.177 \\
0\end{array}$ & $\begin{array}{c}1.281 \\
0.8\end{array}$ & $\begin{array}{c}1.300 \\
1.0\end{array}$ \\
\hline $\begin{array}{l}\text { Scheletro intero } \\
\mathrm{g} / \mathrm{cm}^{2} \\
\mathrm{~T} \text {-score }\end{array}$ & & $\begin{array}{c}0.886 \\
-\end{array}$ & & & $\begin{array}{c}1.101 \\
-0.4\end{array}$ & $\begin{array}{c}1.069 \\
-0.5\end{array}$ & $\begin{array}{c}1.074 \\
-0.5\end{array}$ \\
\hline
\end{tabular}

\section{DISCUSSIONE}

La terapia con vitamina $\mathrm{D}$ e fosfati nel bambino con rachitismo ipofosforemico è codificata da oltre 20 anni da linee guida internazionali e ha lo scopo di correggere e prevenire le deformità scheletriche migliorando l'out- come staturale. Nel paziente adulto invece non esiste ancora una terapia standard condivisa ed è controverso se sia opportuno proseguire la terapia impostata in età giovanile. Da un lato evidenze istologiche mostrano una recrudescenza di osteomalacia nell'adulto dopo l'interruzione di fosforo e calcitriolo (23), dall'altro la maggior parte dei pazienti non presenta disturbi significativi. La 
terapia inoltre predispone al rischio di nefrocalcinosi, calcolosi renale e insufficienza renale (24). L'atteggiamento che spesso prevale è perciò quello di mantenere la terapia fino alla fine della crescita per poi interromperla quando le epifisi sono chiuse.

Dopo la fine della crescita, l'obiettivo terapeutico diventa contrastare l'astenia, i dolori ossei, la perdita di massa ossea e l'osteomalacia. Tuttavia è difficile definire criteri sicuri di riferimento e la dose di $1,25(\mathrm{OH})_{2} \mathrm{D}$ e fosfati capace di raggiungere tali obiettivi deve essere perciò ricercata di volta in volta in ciascun soggetto. La terapia con fosfato non riesce infatti a garantire normali livelli di fosforemia se non per brevi periodi di tempo. Essa però stimola la produzione di PTH ed espone al rischio di iperplasia paratiroidea nonostante il trattamento con calcitriolo (20). Iperparatiroidismo secondario viene infatti riscontrato nella maggior parte dei pazienti dopo alcuni anni di terapia ed è stato attribuito alla formazione di complessi circolanti di calcio-fosfato, che causano una ipocalcemia intermittente e condizionano una persistente stimolazione della ghiandola paratiroidea. E stato inoltre evidenziato che l'endopeptidasi di membrana PHEX è abbondantemente espressa nel tessuto paratiroideo. Le mutazioni inattivanti del gene $P H E X$ potrebbero perciò condurre a una ridotta degradazione della molecola all'interno della paratiroide stessa, causando una sovraproduzione di PTH nei pazienti con rachitismo ipofosforemico X-linked (25).

Come nel dializzato, la storia clinica di questi pazienti è perciò condizionata dallo sviluppo di iperparatiroidismo secondario e dal rischio di una progressiva autonomia secretiva paratiroidea che può sfociare in nodularità ghiandolare e nello sviluppo di adenomi paratiroidei e iperparatiroidismo primitivo $(21,22)$. È questo il caso della paziente da noi seguita, che dopo un lungo periodo di iperparatiroidismo secondario ha sviluppato un adenoma paratiroideo. La terapia standard è stata in lei diversamente modulata nel corso degli anni per limitare lo stimolo alla secrezione di PTH, ma non è stato comunque possibile evitare lo sviluppo di iperparatiroidismo. Nella fase di iperparatiroidismo autonomo, l'ipercalcemia era moderata, la calciuria normale e nonostante la storia clinica, non c'era tendenza alla demineralizzazione ossea alla DXA. A tale riguardo bisogna però considerare che nella osteomalacia il tessuto osteoide esuberante può mascherare un deficit di massa minerale e la DXA non è perciò la metodica ideale per valutare la densità ossea in questi pazienti. Questi pazienti si giovano della paratiroidectomia quale trattamento dell'adenoma paratiroideo, ma la nostra paziente, non tollerando l'idea di un nuovo intervento, è stata da noi indirizzata alla terapia con calciomimetico (26). Questo farmaco si è dimostrato effi- cace nel controllo della calcemia, ma meno efficace sul PTH che si è normalizzato solo nei primi quattro mesi di terapia. In maniera superiore all'atteso, la fosforemia aumentava significativamente durante terapia con cinacalcet.

Aumentando la sensibilità del calcium-sensing receptor al calcio circolante, il cinacalcet riduce la secrezione di PTH e conseguentemente le perdite renali di fosfato. Questo spiega l'incremento della fosforemia, nonostante il deficit di riassorbimento imposto dal difetto renale. Nei pazienti dializzati la valutazione ecografica ha evidenziato come le ghiandole di piccole dimensioni possano decrescere di volume dopo due anni di terapia con cinacalcet (27). Nella nostra paziente non abbiamo assistito a un effetto sul volume ghiandolare, però le calcemie fino a dieci mesi dalla sospensione del cinacalcet erano mediamente più basse di quelle rilevate prima della sua assunzione. Questo suggerisce una attenuazione funzionale della ghiandola paratiroidea che andrà ovviamente confermata con ulteriori dati. Questo comportamento suggerisce anche la possibilità di un uso ciclico del farmaco in questi pazienti. Dati preliminari su 8 bambini con rachitismo ipofosforemico X-linked mostrano che il cinacalcet, in aggiunta alla terapia standard, può prevenire lo sviluppo di iperparatiroidismo secondario (26) e ridurre la secrezione di PTH in risposta a un bolo di fosfato (28). La terapia con cinacalcet potrebbe perciò essere utilizzata nei pazienti con iperparatiroidismo secondario e valori di calcemia normale-alta perché permette di ridurre la quantità di fosfati e vitamina $\mathrm{D}$ somministrati e di diminuire così il rischio di nefrocalcinosi (26).

In conclusione, questo caso clinico conferma che l'iperparatiroidismo è una complicanza del trattamento del rachitismo ipofosforemico e indica nel calciomimetico un utile presidio per la sua gestione in questi pazienti (26), sia per controllare la calcemia nelle forme chiaramente primitive sia perché potrebbe giovare al controllo della fosforemia nelle forme secondarie con basso grado di autonomia secretiva paratiroidea. Questo caso indica anche la necessità di interrompere $\mathrm{o}$ limitare il trattamento con fosfato alla fine della crescita per evitare di mantenere uno stimolo cronico sulla paratiroide. Viceversa i pazienti potrebbero trarre vantaggio dalla terapia con calcitriolo che, oltre a controllare la secrezione di PTH, potrebbe proteggere dalla osteomalacia grazie alla sua azione favorevole sull'assorbimento intestinale del fosfato. 


\section{Riassunto}

Il rachitismo ipofosforemico comprende un gruppo di rare patologie ereditarie caratterizzate da un deficit di riassorbimento renale del fosfato, ipofosfatemia e deformazioni ossee. Le forme più comuni sono il rachitismo ipofosforemico a trasmissione X-linked dominante e il rachitismo ipofosforemico autosomico dominante (AD). Il caso che vi proponiamo è quello di una donna con una diagnosi clinica e biochimica di rachitismo ipofosforemico. La nostra discussione verterà sull'uso della terapia con vitamina $\mathrm{D}$ e fosfati che, nel bambino è codificata da oltre 20 anni e ha lo scopo di correggere e prevenire le deformità scheletriche, mentre nel paziente adulto non è uno standard condiviso ed è controverso se sia opportuno proseguirla. L'obiettivo terapeutico nell'adulto è quello di contrastare l'astenia, i dolori ossei, la perdita di massa ossea e l'osteomalacia. Tuttavia è difficile definire criteri sicuri di riferimento e la dose di $1,25(\mathrm{OH})_{2} \mathrm{D}$ e fosfati capace di raggiungere tali obiettivi. La terapia con fosfato, inoltre, stimola la produzione di PTH ed espone al rischio di iperplasia paratiroidea. È questo il caso della paziente da noi seguita, che dopo un lungo periodo di iperparatiroidismo secondario ha sviluppato un adenoma paratiroideo. Questo caso clinico conferma che liperparatiroidismo è una complicanza del trattamento del rachitismo ipofosforemico e indica nel calciomimetico un utile presidio per la sua gestione in questi pazienti.

Parole chiave: Rachitismo ipofosforemico, Iperparatiroidismo primitivo, Vitamina $\mathrm{D}$, Cinacalcet

\section{Indirizzo degli Autori:}

Francesco Rainone, MD

Unità di Nefrologia e Dialisi

IRCCS Ospedale San Raffaele

Via Olgettina 60

20132, Milano

rainone.francesco@hsr.it

\section{Bibliografia}

1. DiMeglio LA, White KE, Econs MJ. Disorders of phosphate metabolism. Endocrinol Metab Clin North Am 2000; 29: 591-609.

2. Bielesza B, Klaushoferb K, Oberbauer R. Renal phosphate loss in hereditary and acquired disorders of bone mineralization. Bone 2004; 35: 1229-39.

3. Shimada T, Muto T, Urakawa I, et al. Mutant FGF-23 Responsible for autosomal dominant hypophosphatemic rickets is resistant to proteolytic cleavage and causes hypophosphatemia in vivo. Endocrinology 143(8): 3179-82.

4. The ADHR Consortium. Autosomal dominant hypophosphataemic rickets is associated with mutations in FGF23. Nature Genet 2000; 26: 345-8.

5. Lorenz-Depiereux B, Bastepe M, Benet-Page's A, et al. DMP1 mutations in autosomal recessive hypophosphatemia implicate a bone matrix protein in the regulation of phosphate homeostasis. Nature Genet 2006; 38: 1248-50.

6. Lorenz-Depiereux B, Schnabel D, Tiosano D, Hausle G, Strom TM. Loss-of-function ENPP1 mutations cause both generalized arterial calcification of infancy and autosomal-recessive hypophosphatemic rickets. Am J Hum Genet 2010; 86, 267-72.

7. Bergwitz C, Roslin NM, Tieder M, et al. SLC34A3 mutations in patients with hereditary hypophosphatemic rickets with hypercalciuria predict a key role for the sodium-phosphate cotransporter NaPi-IIc in maintaining phosphate homeostasis. Am J Hum Genet 2006; 78:179-92.

8. Magen D, Berger L, Coady MJ, et al. A loss-of-function mutation in NaPi-IIa and renal Fanconi's syndrome. N Engl J Med 2010; 362: 1102-09.

9. Lloyd SE, Pearce SH, Fisher SE, et al. A common molecular basis for three inherited kidney stone diseases. Nature 1996; 379: 445-9.

10. Hoopes RR, Shrimpton AE, Knohl SJ, et al. Dent Disease with mutations in OCRL1. Am J Hum Genet 2005; 76: $260-7$.

11. Lichter-Konecki U, Broman KW, Blau EB, Konecki DS. Genetic and physical mapping of the locus for autosomal dominant renal Fanconi syndrome, on chromosome 15q15.3. Am J Hum Genet 2001; 68: 264-8.

12. Karim Z, Gérard B, Bakouh N, et al. NHERF1 mutations and responsiveness of renal parathyroid hormone. $\mathrm{N}$ Engl J Med 2008; 359: 1128-35.

13. Malloy PJ, Hochberg Z, Tiosano D, Pike JW, Hughes MR, Feldman D. The molecular basis of hereditary 1,25-dihydroxyvitamin D3 resistant rickets in seven related families. J Clin Invest 1990; 86: 2071-9.

14. Cheng, J. B., Levine, M. A., Bell, N. H., Mangelsdorf, D. J., Russell, D. W. Genetic evidence that the human CYP2R1 enzyme is a key vitamin D 25-hydroxylase. Proc Nat Acad Sci 2004; 101: 7711-5. 
15. Hughes MR, Malloy PJ, Kieback DG, et al. Point mutations in the human vitamin $\mathrm{D}$ receptor gene associated with hypocalcemic rickets. Science 1988; 242: 1702-05.

16. Priè D, Frielander G. Reciprocal control of 1,25-dihydroxyvitamin D and FGF23 formation involving the FGF23/Klotho system. Clin J Am Soc Nephrol 2010; 5: $1717-22$.

17. Makras P, Neveen A, Kant SG, Papapoulos SE. Normal Growth and Muscle Dysfunction in X-Linked Hypophosphatemic Rickets Associated with a Novel Mutation in the PHEX Gene. J Clin Endocrinol Metab 2008; 93: 1386-9.

18. Mäkitie O, Doria A, Kooh SW, Cole WG, Daneman A, Sochett E. Early treatment improves growth and biochemical and radiographic outcome in X-linked hypophosphatemic rickets. J Clin Endocrinol Metab 2003; 88: 3591-7.

19. Alon U, Newsome H Jr, Chan JC. Hyperparathyroidism in patients with X-linked dominant hypophosphatemic rickets - application of the calcium infusion test as an indicator for parathyroidectomy. Int J Pediatr Nephrol 1984; 5: 39-43.

20. Mäkitie O, Kooh SW, Sochett E. Prolonged high-dose phosphate treatment: a risk factor for tertiary hyperparathyroidism in X-linked hypophosphatemic rickets. Clin Endocrinol (Oxf) 2003; 58: 163-8.

21. Thomas WC, Fry RM. Parathyroid adenomas in chronic rickets. Am J Med 1970; 49: 404-07.

22. Knudtzon J, Halse J, Monn E, et al. Autonomous hyperparathyroidism in X-linked hypophosphatemic. Clin Endocrinol 1995; 42: 199-203.

23. Marie PJ, Glorieux FH. Relation between hypomineralized periosteocytic lesions and bone mineralization in vitamin D-resistant rickets. Calcif Tissue Int 1983; 35: 443.

24. Vaisbich MH, Koch VH. Hypophosphatemic rickets: results of a long-term follow-up. Pediatr Nephrol 2006; 21: 230-4.

25. Blydt-Hansen TD, Tenenhouse HS, Goodyer P. PHEX expression in parathyroid gland and parathyroid hormone dysregulation in X-linked hypophosphatemia. Pediatr Nephrol 1999; 13: 607-11.

26. Alon US, Levy-Olomucki R, Moore WV, Stubbs J, Quarles LD. Calcimimetics as an adjuvant treatment for familial hypophosphatemic rickets. Clin J Am Soc Nephrol 2008; 3: 658-64.

27. Meola M, Petrucci I, Barsotti G. Long-term treatment with cinacalcet and conventional therapy reduces parathyroid hyperplasia in severe secondary hyperparathyroidism. Nephrol Dial Transplant 2009; 24: 982-9.

28. Patriquin $\mathrm{H} \&$ Robitaille P. Renal calcium deposition in children: sonographic demonstration of the Anderson-Carr progression. Am J Roentgenology 1986; 146: 1253-6.

\section{TEST di VERIFICA - 2}

\section{1) Le forme più comuni di rachitismo ipofosfore- mico sono?}

a) A trasmissione autosomica dominante e $\mathrm{X}$-linked dominante.

b) A trasmissione autosomica recessiva e autosomica dominante.

c) A trasmissione X-linked recessiva e autosomica dominante.

d) A trasmissione mitocondriale.

2) La terapia del rachitismo ipofosforemico prevede, nel bambino:

a) Calcitriolo e chelanti del fosforo.

b) Vitamina B12 e fosfati.

c) Calcitriolo e fosfati.

d) Calcimimetico e fosfati.

3) Nel rachitismo ipofosforemico la protratta terapia con fosfati può predisporre a:
a) Nefrocalcinosi.
b) Iperparatiroidismo.
c) Calcolosi.
d) Tutte le precedenti.

4) Una possibile alternativa terapeutica alla paratiroidectomia per l'iperparatiroidismo è rappresentata da:
a) Calcimimetico.
b) Teriparatide.
c) Lantanio carbonato.
d) Sevelamer.

Le risposte corrette alle domande sono a pag. 18 